\title{
Ballistic Range Testing Data Analysis of Tianwen-1 Mars Entry Capsule
}

\author{
Haogong Wei $\mathbb{D},{ }^{1} \mathrm{Xin} \mathrm{Li}^{2}{ }^{\mathrm{Jie}} \mathrm{Huang},{ }^{2} \mathrm{Qi} \mathrm{Li}^{1}{ }^{1}$ and Wei Rao ${ }^{1}$ \\ ${ }^{1}$ Beijing Institute of Spacecraft System Engineering, Beijing, China \\ ${ }^{2}$ China Aerodynamics Research and Development Center, Mianyang, Sichuan, China \\ Correspondence should be addressed to Haogong Wei; weihaogong@aliyun.com
}

Received 22 July 2021; Accepted 11 November 2021; Published 3 December 2021

Copyright (c) 2021 Haogong Wei et al. Exclusive Licensee Beijing Institute of Technology Press. Distributed under a Creative Commons Attribution License (CC BY 4.0).

\begin{abstract}
A typical blunt body such as Tianwen-1 Mars entry capsule suffers dynamic instability in supersonic regime. To investigate the unstable Mach range of flight and to confirm the design of aerodynamic shape and mass properties, a ballistic range test was carried out aiming at capturing supersonic dynamic characteristics of Tianwen-1. Aerodynamic coefficients of free-flight scaled models were derived by modified linear regression method based on position and attitude data, while the dynamic coefficients were established under the assumption of small angle linearization. The static moment coefficients and dynamic derivatives were identified thereafter. Results show that models in untrimmed configuration are dynamically unstable at certain Mach numbers, whereas models in trimmed configuration are dynamically stable at other Mach numbers tested. Both trimmed and untrimmed configurations are statically stable in all testing cases.
\end{abstract}

\section{Introduction}

The Tianwen-1 Mars entry capsule successfully landed on the surface of Mars in southern Utopia planitia on May 14th, 2021 at 23:18 UTC. Launched aboard CZ-5B from WenChang on July 23th, 2020, the Tianwen-1 Mars exploration mission aims at orbiting, landing, and roving in one trip. The Tianwen-1 spacecraft was injected into Mars orbit in February, 2021, and stayed two and a half months in orbit for optical observations of the landing site before the final touchdown.

Tianwen-1 was programmed to unfold a trim tab at Mach 2.8 to trim the angle of attack (AoA) towards $0^{\circ}$ before parachute deployment at Mach 1.8. A free-flight ballistic range test was conducted in order to obtain the static and dynamic aerodynamic characteristics of Tianwen-1 in trimmed and untrimmed configurations under typical supersonic conditions and to verify the numerical calculation results of supersonic static and dynamic aerodynamic characteristics of the capsule.

Transonic and supersonic dynamic characteristics of blunt body entry vehicles are difficult to calculate by numerical methods, since transient and unsteady flow phenomena such as separation, reattachment, wake, and time-delay are hard to capture accurately. Therefore, researchers prefer studying flight dynamics via ground testing methods. There are three types of tests, i.e., forced oscillation, free oscillation, and free-flight. Blunt bodies, such as Tianwen-1 Mars entry capsule itself, are sensitive to disturbances in transonic and supersonic flows.

It is difficult to capture accurate dynamic characteristics by forced oscillation tests as this method induces considerable disturbance to the flow field unavoidably. The free oscillation method can only be used to obtain the dynamic response in a single degree of freedom, which is considered as a simplified free-flight method. The free-flight method retains all six degrees of freedom of the model under no external disturbance after launching, which reflects the real dynamic characteristics of the model [1].

Chapman et al. studied the limit cycle analysis method of general blunt bodies, and derived the limit cycle expression for different cases [2]. Chapman et al. completed the dynamic stability test of the Stardust sample return capsule in the Aeroballistic Research Facility at Eglin Air Force Base (ARFAFB) and obtained the data from Mach 1.2 to 2.8. It is found that the limit cycle phenomenon is caused by the high nonlinearity of pitch damping and angle of attack [3]. Cheatwood et al. completed the dynamic stability test of 
the Genesis sample return capsule at ARF and found that the shape is dynamically unstable in the range of Mach 1.0 to 4.5 , and the angle of attack range of dynamic unstable expands as Mach number decreasing [4]. Kiritani et al. analyzed the transonic flow field of the Hayabusa sample return capsule from Mach 0.77 to 1.52 using the ballistic range test equipment at Tohoku University [5]. Schoenberger et al. obtained static and dynamic aerodynamic characteristics of the Mars Pathfinder entry capsule from Mach 1.5 to 3.5 at ARFAFB [6]. Brown et al. carried out the free-flight ballistic range test of the Mars Science Laboratory entry capsule [7]. Schoenberger et al. completed the ballistic range test of the Perseverance Mars entry capsule at the Aberdeen Test Center in Maryland and obtained data in support of the second generation of Mars Entry Descent and Landing Instrumentation (MEDLI 2) [8]. Murman et al. carried out the forced oscillation, free oscillation, and free-flight simulation analyses of the general blunt bodies and investigated the nonlinear coupling characteristics [9]. Song et al. studied the motion and aerodynamic characteristics of Soyuz-like capsule with large blunt nose and small lift-drag ratio during free flight in transonic flow [10].

In this paper, a study in dynamic stability of Tianwen-1 entry capsule is discussed regarding the special application of a trim table A typical blunt body such as Tianwen-1 Mars entry capsule suffers dynamic instability in supersonic regime. To investigate the unstable Mach range of flight and to confirm the design of aerodynamic shape and mass properties, a ballistic range test was carried out aiming at capturing supersonic dynamic characteristics of Tianwen- 1 . The position and attitude data was obtained by binocular vision technique, from which the aerodynamic coefficients were derived from. Static and dynamic coefficients are established under the assumption of small angle linearization. The supersonic aerodynamic characteristics of the entry capsule in trimmed and untrimmed configurations are studied in the end.

\section{Test Description}

The tests were carried out in the $200 \mathrm{~m}$ Free-Flight Ballistic Range of China Aerodynamics Research and Development Center. The test medium in the chamber was air. The binocular measurement stations were installed besides the room along the model flying direction, which would be calibrated and aligned to the global base reference coordinate system before the test. There are two configurations of scaled test models: trimmed (with trim tab deployed) and untrimmed (with trim tab folded). The reduce frequency of the oscillating model should be consistent with that in actual flight, i.e., $\oplus_{m}=\oplus_{r}$, where

$$
\bar{\omega}_{i}=\frac{\omega_{i} D}{V}=\sqrt{\frac{m_{i}^{\alpha} \rho V^{2} S D}{2 I_{i}}} \cdot \frac{D}{V}=\sqrt{-\frac{\pi m_{i}^{\alpha} \rho D^{5}}{8 I_{i}}},
$$

where $\bar{\omega}_{i}$ is the reduce frequency, $\omega_{i}$ is the original frequency, $D$ is the diameter, $V$ is the velocity, $m_{i}^{\alpha}$ is static momentum coefficient, $\rho$ is the density, $S=\pi \cdot(D / 2)^{2}$ is the area, and $I_{i}$ is the inertia.

The two configurations of the scaled models are shown in Figure 1. When the flying model with coded marking points on surface entered the measurement field, it would be illuminated by the extended laser beam with the pulse width of smaller than $10 \mathrm{~ns}$; meanwhile, the two images of the target were obtained by the cameras [11]. Mass properties are shown in Table 1. The ratio of momenta of three axes is consistent with that of the actual capsule. However, the nominal mass of these scaled models is designed much heavier than it should be to retain altitude and to produce more data in one shot. If a lighter mass is adopted, the model will lose altitude quickly in flight and thus cannot reach the end of the testing chamber. Less data will be acquired if fewer cameras are passed. Small variations due to manufacture could be noticed in mass properties.

Two typical free-flight test cases are shown in Table 2, with one trimmed and the other not. The test conditions of the two cases were selected based on nominal trajectory, namely, HX01 is aiming at Mach 1.5 average and HX02 at Mach 2.5. The average speed is computed here only for reference purpose. The initial velocity is higher than this average to compensate speed loss due to drag. Density is determined according to reduce frequency. Pressure is governed by density and temperature. The release angle of attack is set to 5 degree for an initial turbulence.

\section{Methods}

The supersonic aerodynamics of the capsule in the free flight is characterized as follows: the weak nonlinearity of the static stability derivative coefficient can be identified by an aerodynamic model which is dominated by linear term $\left(\mathrm{C}_{m q}+\mathrm{C}_{m \dot{\alpha}}\right)_{0}$ at small angle of attacks and by nonlinear term $\left(\mathrm{C}_{m q}+\mathrm{C}_{m \dot{\alpha}}\right)_{2} \alpha^{2}$ at high angle of attacks. The range of angle of attack oscillation is within $\pm 10^{\circ}$ in most cases; thus, the linearization assumption for small angle of attack is applicable.

By minimizing the error variance sum of each motion equation, the corresponding aerodynamic coefficients can be obtained. The aerodynamic coefficients cannot be measured directly by flight test and need to be calculated according to the acceleration measurements

$$
\begin{aligned}
& C_{x}=\frac{m a_{x}}{\bar{q} S}, \\
& C_{y}=\frac{m a_{y}}{\bar{q} S}, \\
& C_{z}=\frac{m a_{z}}{\bar{q} S},
\end{aligned}
$$

where $C_{x}$ is the axial force coefficient, $C_{y}$ is the normal force coefficient, and $C_{z}$ is the lateral force coefficient; $m$ is the mass, $a_{x}, a_{y}$, and $a_{z}$ are accelerations in three axes, $\bar{q}$ is the dynamic pressure, $S$ is the reference area, and $l$ is the reference length. After the sensor error was corrected by the 


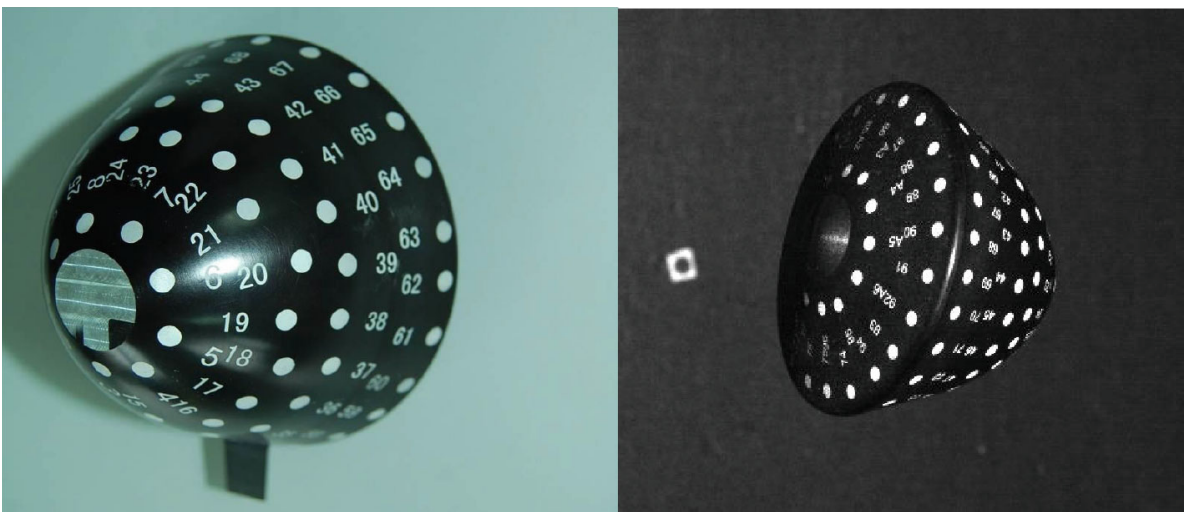

FIGURE 1: Scaled model of the capsule with coded marking points (left: trimmed; right: untrimmed).

TABLE 1: Mass properties (partial).

\begin{tabular}{lccccccc}
\hline Shot & Model & $\begin{array}{c}\text { Mass } \\
(\mathrm{g})\end{array}$ & $\begin{array}{c}\text { Diameter } \\
(\mathrm{mm})\end{array}$ & $\begin{array}{c}\text { Length } \\
(\mathrm{mm})\end{array}$ & $\begin{array}{c}\text { Roll momentum } \\
\left(\mathrm{g} \cdot \mathrm{cm}^{2}\right)\end{array}$ & $\begin{array}{c}\text { Yaw momentum } \\
\left(\mathrm{g} \cdot \mathrm{cm}^{2}\right)\end{array}$ & $\begin{array}{c}\text { Pitch momentum } \\
\left(\mathrm{g} \cdot \mathrm{cm}^{2}\right)\end{array}$ \\
\hline HX01 & Trimmed & 451.96 & 100.00 & 76.66 & 5009.71 & 3756.75 & 4036.43 \\
HX02 & Untrimmed & 454.90 & 99.98 & 76.68 & 5018.13 & 3799.07 & 4002.96 \\
\hline
\end{tabular}

TABle 2: Test cases (partial).

\begin{tabular}{lccccccc}
\hline Shot & Model & Mass $(\mathrm{g})$ & Average speed $(\mathrm{m} / \mathrm{s})$ & Pressure $(\mathrm{kPa})$ & Temperature $\left({ }^{\circ} \mathrm{C}\right)$ & Mach & Release AoA $\left({ }^{\circ}\right)$ \\
\hline HX01 & Trimmed & 451.96 & 500 & 14.940 & 13.7 & 1.47 & 5 \\
HX02 & Untrimmed & 456.30 & 848 & 2.582 & 25.0 & 2.45 & 5 \\
\hline
\end{tabular}
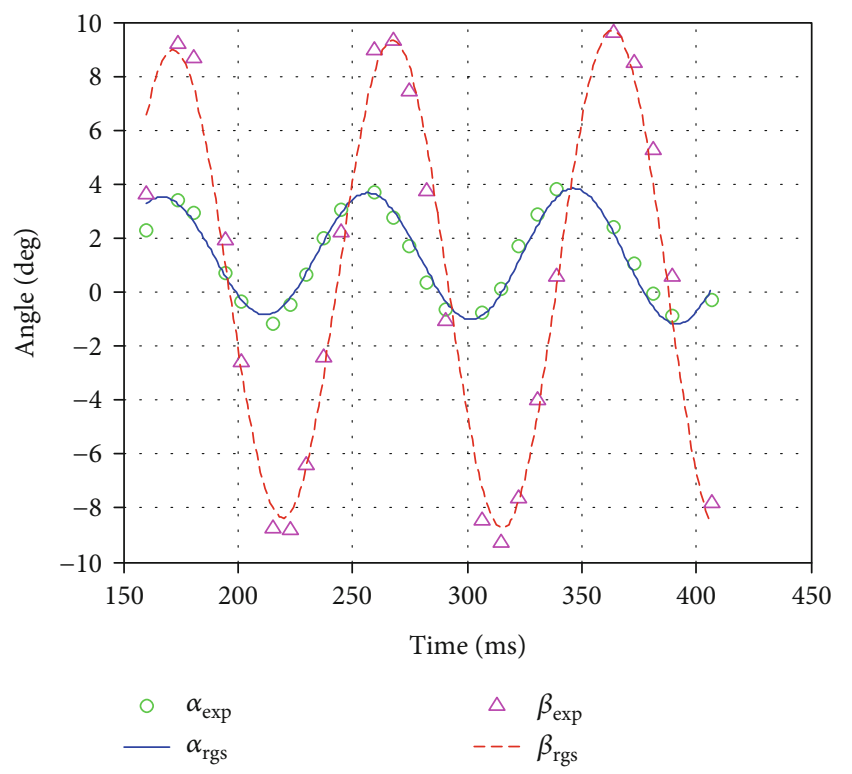

FIGURE 2: Angle of attack and sideslip of the model with trim tab (Shot HX01). trajectory reconstruction, the aerodynamic coefficients can be calculated. The lift coefficient $C_{L}$ and drag coefficient $C_{D}$ can be converted from the following equations:

$$
\begin{aligned}
& C_{L}=C_{y} \cos \alpha-C_{x} \sin \alpha, \\
& C_{D}=C_{y} \sin \alpha+C_{x} \cos \alpha .
\end{aligned}
$$

Because the products of inertia cannot be measured directly, the definition of the body axes should be coincide with the inertial principal axes of the vehicle, so that the products of inertia are $I_{x y}=I_{y z}=I_{z x}=0$. The products of inertia are considered ignorable in engineering practice and retain sufficient accuracy if the angle between the body $x$-axis and the true $x$-axis of inertia align with $I_{x}$ is less than one degree. The total moment coefficients can be expressed as

$$
\begin{aligned}
& C_{m x}=\frac{1}{\bar{q} S l}\left[I_{x} \dot{\omega}_{x}+\left(I_{z}-I_{y}\right) \omega_{z} \omega_{y}\right], \\
& C_{m y}=\frac{1}{\bar{q} S l}\left[I_{y} \dot{\omega}_{y}+\left(I_{x}-I_{z}\right) \omega_{x} \omega_{z}\right], \\
& C_{m z}=\frac{1}{\bar{q} S l}\left[I_{z} \dot{\omega}_{z}+\left(I_{y}-I_{x}\right) \omega_{y} \omega_{x}\right],
\end{aligned}
$$




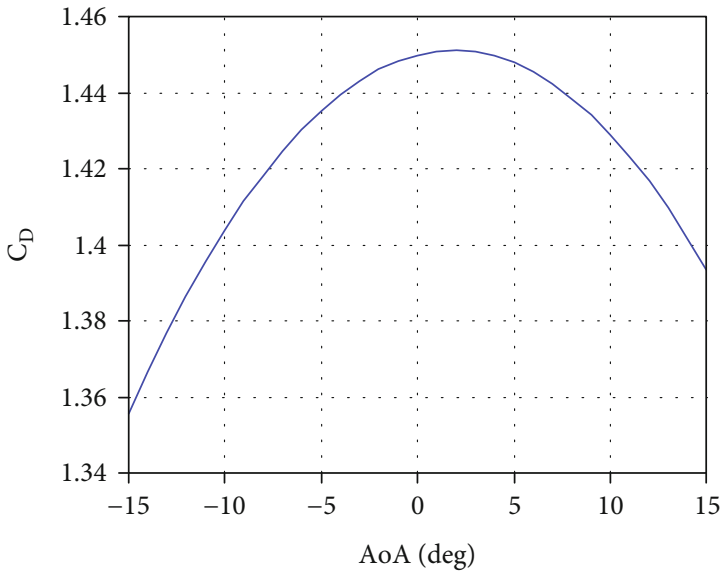

(a) Drag coefficient

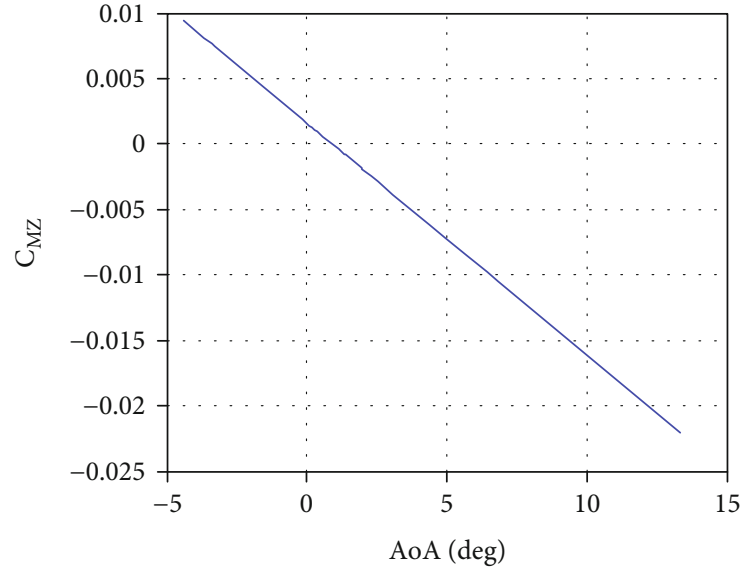

(b) Pitching moment $-M=1.5$

FIGURE 3: Static aerodynamic coefficients of free-flight model in trimmed configuration.

where $C_{m x}$ is the rolling moment coefficient, $C_{m y}$ is the yawing moment coefficient, $C_{m z}$ is the pitching moment coefficient, and $I_{x}, I_{y}$, and $I_{z}$ are the three-axis inertia, respectively. The aerodynamic moment coefficients cannot be measured directly and need to be calculated according to the measured value of the angular velocity.

The aerodynamic model of the vehicle assumes that the aerodynamic parameters are functions of the flight state parameters and control inputs. Let the aerodynamic parameters $C_{x}, C_{y}, C_{z}, C_{m x}, C_{m y}$, and $C_{m z}$ be the aerodynamic forces and moments on the system axes, respectively, and let the flight state parameters and control inputs be $x=$ $\left(x_{1}, x_{2}, \cdots, x_{n}\right)^{T}$. Taking the aerodynamic moment coefficient $C_{m z}(t)$ as an example, the general form of the mathematical model of the aerodynamic parameters is as follows,

$$
C_{m z}(t)=\sum_{i_{1}=0}^{N_{1}} \cdots \sum_{i_{p}=0}^{N_{p}} C_{m z}^{x^{\pi i} k} \prod_{k=1}^{p} x_{k}^{i_{k}}(t)
$$

where $x_{k}$ is one of the flight state parameters $(k=1,2, \ldots, n)$, such as $\alpha, \beta, \omega_{z}, \ldots, \delta_{z}$, and $i_{k}$ is the power of $x_{k}$, and $C_{m z}^{x^{\pi i k}}$ is the derivative of $C_{m z}$ corresponding to $\prod_{k=1}^{p} x_{k}^{i_{k}}(t)$, where $i_{k}$ $=0,1, \ldots, N_{k}$. Usually, each term is the product of 1 to 3 state parameters, and the highest power is thus normally taken as 3 or 4 , except for a few individual terms that can be selected with higher powers based on a priori knowledge, theoretical calculations, or ground test results.

The six-degree-of-freedom dynamic equation is linearized in plane, and the force and moment are linearized with small angle assumption [6].

$$
\ddot{\alpha}-\frac{\rho V S}{2 m}\left(-C_{L \alpha}+\left(C_{m q}+C_{m \dot{\alpha}}\right) \frac{D^{2}}{2 \sigma^{2}}\right) \dot{\alpha}-\frac{\rho V^{2} S D}{2 I} C_{m \alpha} \alpha=0,
$$

TABle 3: Static and dynamic moment derivatives of model in trimmed configuration.

\begin{tabular}{lcccc}
\hline & Pitch & & & Yaw \\
$C_{m z}^{\alpha}$ & $C_{m z}^{\omega_{z}}$ & $C_{m y}^{\beta}$ & & $C_{m y}^{\omega_{y}}$ \\
\hline-0.113 & 1.28 & -0.092 & & 0.292 \\
\hline
\end{tabular}

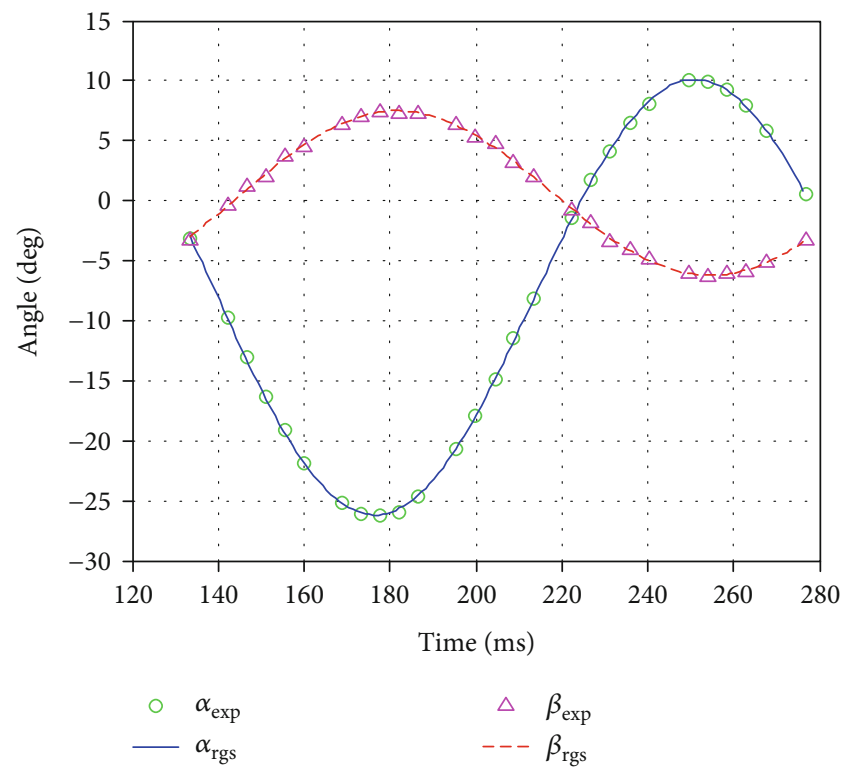

FIgURE 4: Results of angle of attack and sideslip of untrimmed configuration (Shot HX02).

where for the coefficient of constant term, the solution of the above equation is in damped resonant form.

$$
\alpha(t)=\alpha_{0} e^{\gamma t} \cos (\omega t),
$$




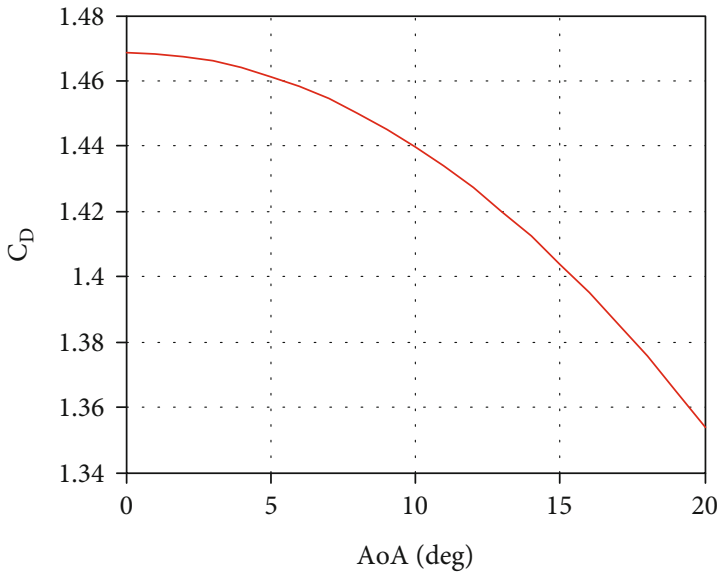

(a) Damping coefficient

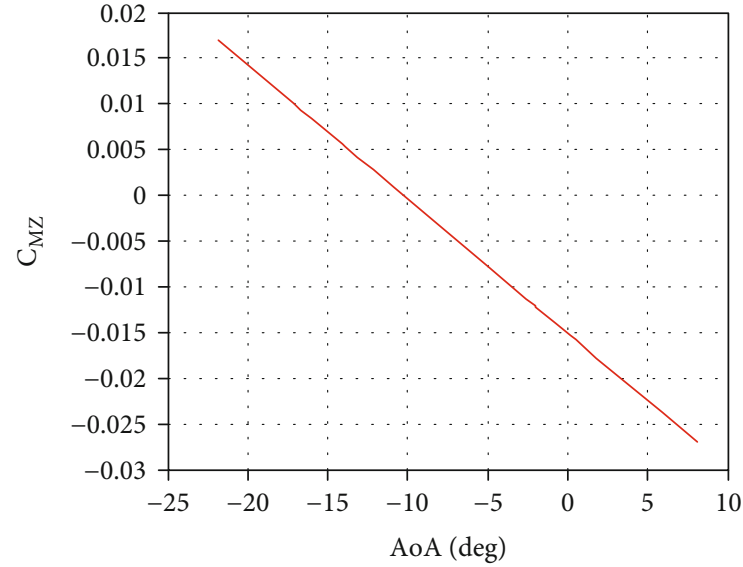

(b) Pitching moment $M=2.5$

FIgURe 5: Aerodynamic coefficient curve of free flight of the untrimmed configuration.

where

$$
\begin{gathered}
\gamma=\frac{\rho V S}{4 m}\left(-C_{L \alpha}+\left(C_{m q}+C_{m \dot{\alpha}}\right) \frac{D^{2}}{2 \sigma^{2}}\right), \\
\omega=\sqrt{\frac{\rho V^{2} S D}{2 I} C_{m \alpha}-\gamma^{2},}
\end{gathered}
$$

where in equation (6), $\gamma$ is the damping term and dominated by $\left(C_{m q}+C_{m \dot{\alpha}}\right)$. When $\gamma$ is positive (so that $\left(C_{m q}+C_{m \dot{\alpha}}\right)$ is positive), the amplitude increases; when $\gamma$ is negative, the amplitude decreases. $\omega$ is the frequency term, which is determined by the square root of the static stability coefficient $C_{m \alpha}$.

It is important to extract static coefficients in order to obtain the dynamic derivative. The lift coefficient can be expressed by the aerodynamic coefficient as

$$
C_{L}=-C_{A} \sin \alpha_{T}+C_{N} \cos \alpha_{T} .
$$

The derivative of $C_{L}$ with respect to $\alpha_{T}$ is written as

$$
C_{L \alpha}=-C_{A} \cos \alpha_{T}-C_{N} \sin \alpha_{T} .
$$

The axial force coefficient and the static stability coefficient are the key parameters to extract the pitching dynamic derivative in combination with equation (6).

\section{Results and Analysis}

4.1. Trimmed Configuration. The angle of attack and sideslip results of shot HX01 (trimmed configuration) are shown in Figure 2. There are diverge oscillations of angle of attack and sideslip, indicating that the trimmed configuration is dynamically unstable in pitch and yaw directions between Mach 1.2 and 1.6.

The results of drag coefficient and pitch moment curve of trimmed configuration in shot HX01 are shown in Figure 3. The maximum drag of Shot HX01, the trimmed configuration, appears at $\mathrm{AoA}=1^{\circ} \sim 2^{\circ}$ Mach 1.5, with the maximum value of 1.45. According to Figure 3(b), the line of pitch moment passes zero at $\mathrm{AoA}=1^{\circ}$, suggesting that there is no pitch moment when $\mathrm{AoA}=1^{\circ}$; hence, the trim angle of attack is $1^{\circ}$. Theoretically, both angle of attack of the maximum drag and zero pitch moment should be $0^{\circ}$. This small deviation in trim angle of attack is caused by two likely reasons. On one hand, the selection of parameters in Eq. (5) affects the result considerably (the parameters are selected cautiously and each with evaluations accordingly). On the other hand, small variations in shape and mass property of the model might exist after manufacturing and assembling, which leads to the deviation in trim angle of attack.

The results of the static and dynamic derivatives of the aerodynamic moment in pitch and yaw directions of the model in trimmed configuration are shown in Table 3 , where $C_{m z}^{\alpha}$ and $C_{m y}^{\beta}$ represent the static derivatives, and $C_{m z}^{\omega_{z}}$ and $C_{m y}^{\omega_{y}}$ the dynamic derivatives, of the pitch and yaw moments relative to the angle of attack and sideslip angle, respectively. It can be seen from Table 3 that the static derivatives are negative in the pitch and yaw directions for the model in trimmed configuration in shot HX01, whereas the dynamic derivatives are positive. In summary, the model in trimmed configuration is statically stable but dynamically unstable at Mach 1.5.

4.2. Untrimmed Configuration. The results of angle of attack and sideslip angle of shot HX02 in untrimmed configuration are shown in Figure 4. It is difficult to evaluate dynamic stability simply from the angular motion history since insufficient period exists during test run.

The drag coefficient and pitch moment curve of the model in untrimmed configuration in shot $\mathrm{HX02}$ are shown in Figure 5. The results show that the maximum axial force on the capsule in untrimmed configuration appears near Mach 2.5 at around $0^{\circ}$ of angle of attack, and the maximum axial force is 1.47; the pitch moment 
TABLE 4: Static and dynamic moment derivatives of the untrimmed configuration.

\begin{tabular}{lcccc}
\hline & Pitch & & \multicolumn{2}{c}{ Yaw } \\
$C_{m z}^{\alpha}$ & $C_{m z}^{\omega_{z}}$ & $C_{m y}^{\beta}$ & $C_{m y}^{\omega_{y}}$ \\
\hline-0.084 & -1.563 & -0.083 & -1.755 \\
\hline
\end{tabular}

passes through zero near $-10^{\circ}$ of angle of attack; hence, the trim angle of attack is $-10^{\circ}$.

The results of the aerodynamic moment static and dynamic derivatives in pitch and yaw directions of the untrimmed configuration are shown in Table 4 , where $C_{m z}^{\alpha}$ and $C_{m y}^{\beta}$ represent the static derivatives of pitch and yaw moment relative to the angle of attack and sideslip, respectively. The results shows that both static and dynamic derivatives of the untrimmed configuration in shot HX02 are negative, which indicates that both pitch and yaw directions of the untrimmed configuration are statically and dynamically stable at Mach 2.45 with small angle of attack.

\section{Conclusion}

The identification algorithm of the aerodynamic parameters for the free-flight ballistic range test is established, and the aerodynamic parameters for the free-flight ballistic range test results of Tianwen-1 Mars entry capsule are completed. The static and dynamic aerodynamic characteristics of the free-flight capsule in both trimmed and untrimmed configurations are acquired under typical supersonic conditions.

Based on analysis of the position and attitude, attitude oscillation, aerodynamic force, static and dynamic stability of the capsule, it is demonstrated that the ballistic range test captures the attitude behaviors and aerodynamic characteristics of Tianwen-1 Mars entry capsule. The results of the pitch and yaw moment coefficients exhibit the aerodynamic characteristics of the capsule. The capsule in trimmed configuration is dynamically unstable in the pitch and yaw directions, whereas the untrimmed configuration is dynamically stable. In both cases, the capsule is statically stable in pitch and yaw directions.

\section{Data Availability}

The experimental data used to support the findings of this study are available from the corresponding author upon request.

\section{Conflicts of Interest}

The authors declare that there are no conflicts of interest regarding the publication of this article.

\section{References}

[1] D. Bogdanoff, Design guide for aerodynamics testing of earth and planetary entry vehicles in a ballistic range, 2017, NASA/ TM-2017-219473.
[2] G. Chapman and L. Yates, "Limit Cycle Analysis of Planetary Probes," in 37th AIAA Aerospace Sciences Meeting and Exhibit, Reno, NV, 1999.

[3] G. Chapman, R. Mitcheltree, and W. Hathaway, "Transonic and low supersonic static and dynamic aerodynamic characteristics of the Stardust sample return capsule," in 37th Aerospace Sciences Meeting and Exhibit, Reno, NV, 1999.

[4] F. Cheatwood, G. Winchenbach, W. Hathaway, and G. Chapman, "Dynamic stability testing of the genesis sample return capsule," in 38th Aerospace Sciences Meeting and Exhibit, Reno, NV, 2000.

[5] H. Kiritani, N. Tanaka, K. Ohtani, K. Fujita, and H. Nagai, "Transonic flow field analysis of a free-flight capsule using ballistic range," in AIAA Scitech 2020 Forum, Orlando, FL, 2020.

[6] M. Schoenenberger, W. Hathaway, L. Yates, and P. Desai, "Ballistic Range Testing of the Mars Exploration Rover Entry Capsule," in 43rd AIAA Aerospace Sciences Meeting and Exhibit, Reno, NV, 2005.

[7] J. Brown, L. Yates, D. Bogdanoff, G. Chapman, M. Loomis, and T. Tam, "Free-flight testing in support of the Mars science laboratory aerodynamics database," Journal of Spacecraft and Rockets, vol. 43, no. 2, pp. 293-302, 2006.

[8] M. Schoenenberger, G. Brown, and L. Yates, "Surface pressure ballistic range test of Mars 2020 capsule in support of MEDLI2," in 35th AIAA Applied Aerodynamics Conference, Denver, Colorado, 2017.

[9] S. Murman and M. Aftosmis, "Dynamic Analysis of Atmospheric-Entry Probes and Capsules," in 45th AIAA Aerospace Sciences Meeting and Exhibit, Reno, NV, 2007.

[10] W. Song, B. Ai, Z. Jiang, and W. Lu, "Free-flight static and dynamic aerodynamic characteristics for re-entry capsule at transonic speed," Journal of Experiments in Fluid Mechanics, vol. 33, no. 4, pp. 89-94, 2019.

[11] F. Ke, J. Huang, X. Li et al., "Vision measurement technique of model position and its widespread application on the ballistic range," Measurement, vol. 140, pp. 486-496, 2019. 